Mots. Les langages du politique

\title{
Régularités et répartition. Analyse de quelques métaphores dans L'Express et Le Monde
}

\section{Gunila von Malmborg}

\section{(2) OpenEdition \\ Journals}

Édition électronique

URL : https://journals.openedition.org/mots/1664

DOI : $10.4000 /$ mots. 1664

ISSN : 1960-6001

Éditeur

ENS Éditions

\section{Édition imprimée}

Date de publication : 1 novembre 2005

Pagination : 101-115

ISBN : 2-84788-084-4

ISSN : 0243-6450

\section{Référence électronique}

Gunila von Malmborg, « Régularités et répartition. Analyse de quelques métaphores dans L'Express et Le Monde », Mots. Les langages du politique [En ligne], 79 | 2005, mis en ligne le 28 mai 2008, consulté le 23 avril 2022. URL : http://journals.openedition.org/mots/1664; DOI : https://doi.org/10.4000/mots. 1664

\section{(C) ENS Éditions}




\section{Régularités et répartition. Analyse de quelques métaphores dans L'Express et Le Monde}

Le domaine choisi comme objet d'analyse dans cet article est constitué par les rubriques Politique extérieure et Politique intérieure (dorénavant abrégés Pe et $\mathrm{Pi}$ ) dans une vingtaine de livraisons du Monde et de L'Express (abrégés $\mathrm{M}$ et $\mathrm{X}$ ) des années 1997-1998. Notre but est de faire, à partir de ces journaux, une étude comparative des métaphores appartenant à trois champs génériques ${ }^{1}$ (voir pour ce terme Baylon et Mignot, 1995, p. 116-1202) à savoir Déplacements, Médecine et Phénomènes naturels. Cette étude peut nous fournir de multiples informations: elle permet d'analyser par exemple les rapports entre les deux termes de la métaphore, le terme métaphorisant et le terme métaphorisé. L'inventaire des métaphores nous apprendra aussi comment les métaphores des champs génériques choisis se distribuent sous les deux rubriques et dans les deux journaux. L'observation des termes métaphorisés nous renseignera enfin sur les rapports plus ou moins figés entre les deux parties de la métaphore ainsi que sur la question de savoir s'il existe parmi ces termes des domaines sémantiques ou lexicaux prédominants.

$M$ et $X$ présentent d'importantes similarités quant aux sujets traités. Ils affichent aussi des différences non négligeables, par exemple leur rythme de parution (un quotidien et un hebdomadaire), la longueur des articles (l'hebdomadaire $X$ fait en général apparaître dans le même numéro plusieurs articles sur un seul thème, ce qui ne se rencontre pas dans le quotidien $M$ ), enfin leur profil stylistique ${ }^{3}$. La présence de similarités permettra une certaine généralisation des résultats obtenus, tandis que la présence de différences pourra signaler des traits spécifiques de leur qualité de quotidien et d'hebdomadaire.

Il existe à l'université de Stockholm deux corpus informatisés du $M$ et de $X$, dont le plus récent, COSTO 2, nous a fourni le matériau de nos observations sur la présence des métaphores dans la presse. Il est précédé de COSTO 1, un corpus

1. Le sémanticien François Rastier les appelle domaines.

2. Les références bibliographiques sont développées en fin d'article.

3. Pour la notion de style, nous empruntons la définition de P. Guiraud dans Le Petit Robert (1996, p. 2153) : «L'aspect de l'énoncé qui résulte du choix des moyens d'expression déterminé par la nature et les intentions du sujet parlant ou écrivant. »

Université de Stockholm, Institut de français et d’italien, Suède, guvo8215@student.su.se 
informatisé composé de 13 livraisons du $M$ et du même nombre de livraisons de $X$ parues de mars 1987 à février 1988, époque où il n'existait encore aucun grand corpus journalistique français, même pas en France (Engwall et Bartning, 1989, p. 344). Ce premier corpus de 1987-1988 a été complété par un autre, COSTO 2, de 1997-1998, catégorisé de la même manière que COSTO 1 et également composé de 13 livraisons de chaque journal. On étudiera ici les métaphores dans les articles sous les rubriques Pe et Pi dans COSTO 2. Il sera donc question d'un inventaire des métaphores des trois champs génériques Déplacements, Médecine $^{4}$ et Phénomènes naturels, ces trois champs génériques produisant le plus grand nombre de métaphores dans le corpus examiné (von Malborg, 2004, p. 56). Cet inventaire constituera simultanément une comparaison fréquentielle de ces métaphores dans $M$ et $X$. Les titres, qui peuvent être considérés comme des textes à part entière et dépendant de facteurs exogènes au contenu de l'article proprement dit, par exemple l'organisation typographique de la page (Sullet-Nylander, 1998, p. 22-30), n’ont pas été inclus dans cet examen.

\section{Quelques notions théoriques}

La plupart des linguistes qui se sont penchés sur les caractéristiques de la métaphore semblent d'accord sur un principe de base: il y a incompatibilité sémantique entre le terme métaphorique et son contexte, dont il se démarque. Georges Kleiber parle de "déviance» sémantique et définit cette déviance comme "une transgression de l'usage ordinaire des termes et combinaisons, en somme un 'délit littéral' »(Charbonnel et Kleiber, 1999, p. 102). C'est un critère qui vaut pour toutes les métaphores, depuis celles d'énonciation 5 jusqu'à celles de convention, puisque la déviance concerne la catégorisation : le terme métaphorique exprime une occurrence qui, selon l'usage littéral, ne correspond pas à sa catégorie. Deux facteurs sont donc à l'œuvre quand nous détectons une métaphore : la partie déviante et le contexte dont elle se démarque. Nous appellerons la partie déviante terme métaphorisant. Si ce dernier entre en relation syntaxique avec un syntagme qu'il métaphorise, celui-ci est appelé terme métaphorisé, et la métaphore résultant de cette jonction métaphore combinatoire ${ }^{6}$. À titre d'illustration, voici un exemple :

Depuis, Olivier Schrameck [terme métaphorisé] fait tourner la lourde machine de Matignon. « /l est [...] la véritable tour de contrôle [terme métaphorisant] de la maison », note Manuel Valls7. (X, Pi, 19 février 1998, p. 34-36)

4. Élargissant cette notion, nous y faisons entrer aussi les champs génériques Naissance et Mort.

5. Terme que nous empruntons à Villard (1984, p. 35). D'autres appellations sont par exemple métaphore d'invention, métaphore occasionnelle/nouvelle.

6. Nous empruntons également cette expression à Villard (1984, p. 49).

7. Dans les exemples, le terme métaphorisant est souligné et le terme métaphorisé est en italiques. 
Dans l'exemple ci-dessus, il, anaphore de Olivier Schrameck, est joint au terme métaphorisant par la copule. Les métaphores où il n'existe pas de jonction syntaxique entre les deux termes de la métaphore, où seul le contexte du terme métaphorisant signale la métaphoricité de celui-ci, sont appelées métaphores pragmatiques, étant donné que la syntaxe, et dans un certain nombre de cas la sémantique, ne nous aident pas à interpréter leur message métaphorique, mais seulement le contexte où elles s'insèrent ou les expériences préalables du lecteur.

La métaphore est souvent rapprochée de l'analogie et de la comparaison. Toute interprétation d'une métaphore passe par un raisonnement analogique, où nous utilisons ou inventons des traits qui unissent la métaphore à sa source. L'analogie qui sous-tend la métaphore propose une alternative sémantique (Hallyn, 1994, p. 112), et c'est le sujet parlant qui l'élabore lui-même. Dans l'analogie explicite, d'autre part, deux thèmes sont introduits, privant le sujet parlant de tout espace d'interprétation.

La comparaison ressemble à l'analogie en ce que les deux termes, thème et phore pour l'analogie, comparé et comparant pour la comparaison, sont présents. De plus, la comparaison relève d'une logique du vrai et du faux, ce qui n'est pas le cas pour la métaphore (Charbonnel et Kleiber, 1999, p. 95). II faut donc écarter l'analogie explicite et la comparaison du domaine de la métaphore qui, elle, pose non une similitude mais une vérité, la vérité expérientielle (Détrie, 2001, p. 273).

\section{Questions de méthode : délimitation des champs génériques; étude pilote}

Dans le but de cerner les champs génériques les plus importants, nous avons repéré toutes les métaphores sous les rubriques concernées dans trois livraisons du $\mathrm{M}$ et de $\mathrm{X}$. Les champs génériques les plus productifs - Déplacements, Médecine et Phénomènes naturels - sont suivis par Arts et Guerre. Notre résultat est conforté par un examen entrepris par Schmitt (1988) dans Le Monde, Le Figaro et L'Humanité, qui place en tête ces trois champs génériques, suivis par Génie militaire, lutte, guerre. Étant donné le temps écoulé depuis l'examen fait par Schmitt, il semble que les champs génériques restent stables pendant longtemps. Les ouvrages plus récents sur la métaphore dans la presse sont plutôt rares. Citons toutefois Kerzazi-Lasri (2003) et von Malmborg (2004) pour la presse française, et Pappas (2003) pour la presse grecque et pour la presse suédoise.

Le lexique de termes métaphorisants obtenu lors de la première lecture des livraisons mentionnées ci-dessus et des articles de Schmitt (1988) a été augmenté à l'aide de synonymes, antonymes, hyponymes, affixes et dérivations. Ainsi, une métaphore contenant le terme métaphorisant bateau a pu être un 
point de départ dans la recherche des métaphores créées à l'aide de termes métaphorisants tels que navire, barque, vaisseau, paquebot. On trouve d'autres métaphores ressortissant au même champ générique, par exemple phare et naviguer. En parcourant notre corpus à la recherche d'exemples de cette première liste, nous en avons croisé d'autres, pertinents par rapport aux champs génériques qui nous intéressent, et qui ont été ajoutés au lexique.

Du choix que nous avons fait, pour notre étude, d'un quotidien et d'un hebdomadaire, pourraient dépendre le caractère plus ou moins stéréotypé, la fréquence et la distribution des métaphores, étant donné que ces journaux diffèrent sensiblement l'un de l'autre par leurs contraintes rédactionnelles. Les conditions temporelles du bouclage des articles, l'intention de couvrir certains domaines de la réalité, la longueur des articles, le profil du lectorat visé sont autant de facteurs pouvant influencer le choix des métaphores.

Notre analyse est divisée en deux parties dont la première est un bref aperçu de la fréquence des termes métaphorisants. Dans un premier temps, chacun des trois champs génériques a été divisé en groupes sémiques, que nous nommons tels étant donné que les lexies dans chaque groupe ont une composante sémique en commun. Le tableau ci-dessous montre comment est faite la division en groupes sémiques et, le cas échéant, en sous-groupes.

\begin{tabular}{|c|c|c|}
\hline \multicolumn{3}{|l|}{ Champ générique } \\
\hline Déplacements & Médecine & Phénomènes naturels \\
\hline $\begin{array}{l}\text { Groupes sémitiques / } \\
\text { sous-groupes }\end{array}$ & Groupes sémitiques & Groupes sémitiques \\
\hline \multirow{2}{*}{$\begin{array}{l}\text { 1. Circulation } \\
\text { a/ Véhicules de terre et leur } \\
\text { équipement }\end{array}$} & $\begin{array}{l}\text { 1. Souffrir de/diagnostiquer } \\
\text { maladie }\end{array}$ & $\begin{array}{l}\text { 1. Climat, météorologie, } \\
\text { sinistres }\end{array}$ \\
\hline & 2. Retour à la santé & 2. Phénomènes célestes \\
\hline \multirow{2}{*}{$\begin{array}{l}\text { b/ Aviation, espace } \\
\text { c/ Navigation }\end{array}$} & $\begin{array}{l}\text { 3. Entrave au bon fonctionne- } \\
\text { ment }\end{array}$ & 3. Aquatique \\
\hline & 4. Affaiblir / faire disparaître & 4. Topographie \\
\hline \multirow{2}{*}{$\begin{array}{l}\text { 2. Déplacements } \\
\text { a/ Pourse déplacer }\end{array}$} & 5. Faire apparaître & 5. Végétal \\
\hline & & \\
\hline \multicolumn{3}{|l|}{ b/ L'action de se déplacer } \\
\hline \multicolumn{3}{|l|}{$\begin{array}{l}\text { 3. Pour faciliter le déplacement } \\
\text { (orientation, signalisation, vitesse) }\end{array}$} \\
\hline 4. / Faire / échouer & & \\
\hline
\end{tabular}

Tableau 1

La division des champs génériques en groupes sémiques 
En scrutant la répartition des terme métaphorisants selon les différents groupes sémiques, nous pourrons par exemple observer comment certains d'eux sont accumulés sous l'une ou l'autre des rubriques examinées. En ce qui concerne les termes métaphorisés, leur identification peut nous montrer les tendances collocantes des métaphores, c'est-à-dire l'uniformité lexico-syntaxique créée par l'usage répété des mêmes lexies dans des constructions syntaxiques identiques.

Comment alors catégoriser les termes métaphorisés ? La dichotomie opérée parFontanier (1977 [1830], p. 103) - qui les classe en «physiques » et «moraux » - nous paraissant trop vague pour notre objectif, nous avons élargi le nombre de catégories, obtenant, là où Fontanier parle de "métaphore physique», une catégorie Concret et une autre Humain. Ce sont des catégories restreintes, à l'encontre de celle, grande et floue, où Fontanier voit des « objets quelconques de la pensée [...] abstraits, moraux ou métaphysiques » (id., p. 101). Il est cependant possible d'en dégager un groupe qui aurait peut-être sa correspondance dans ce que Fontanier caractérise par moral: l'État, les divisions administratives comme par exemple le canton, les entreprises, bref, tous les organismes qui, ultérieurement, sont composés d'individus. Nous appelons ce groupe Collectivité, nous inspirant de la définition du Petit Robert (1996, p. 404) : « 1. Ensemble d'individus groupés naturellement ou pour atteindre un but commun. 2. Circonscription administrative (commune, département, région, etc.) dotée de la personnalité morale.»

Cherchant d'autres critères de délimitation, nous les avons trouvés trop fins ou trop vastes pour notre objectif. Après avoir analysé nos données, nous avons fait une division ultérieure des termes métaphorisés en Abstrait et Immatériel où nous appliquons, pour ceux de la catégorie Immatériel, deux critères, dont un seul peut suffire à assurer la validité du terme :

- les termes métaphorisés sont des lexies dont les référents sont perceptibles par nos sens; quelques exemples en sont pop music, spectacle, des sons;

- les termes métaphorisés sont à ramener à des objets ou des actions concrets.

Le deuxième critère nous permet de ranger un certain nombre d'activités dans cette catégorie, par exemple transaction, perquisitions et d'autres noms ou expressions comme la monnaie unique, style. La division proposée laisse dans la catégorie Abstrait les noms et adjectifs référant à des états psychologiques ou à des comportements comme inquiétude, euphorique, répression, et ceux référant à des idéologies et au monde des idées, tels révolutionnaire, francophonie, surréalisme. Cette division nous sera utile pour dégager des tendances et des thématiques dans le jeu entre termes métaphorisés et termes métaphorisants. 


\section{Récapitulation des fréquences des termes métaphorisants}

\section{Fréquence absolue et relative}

Nous nous pencherons d'abord sur la présence des termes métaphorisants dans les différents groupes sémiques identifiés. Dans les tableaux $2 a$ à $2 \mathrm{C}$ ci-dessous est montré le nombre d'occurrences des termes métaphorisants dans $M$ et $X$ pour chaque groupe sémique ainsi que leur répartition sous les deux rubriques examinées, Pe et Pi. Afin de saisir la variation lexicale dans l'usage des termes métaphorisants, nous avons aussi indiqué le nombre de types utilisé sous les deux rubriques pour chacun des groupes sémiques.

\begin{tabular}{|l|c|c|c|c|c|c|c|}
\hline & \multicolumn{3}{|l|}{ Types/occurrences M } & \multicolumn{3}{|c|}{ Types/occurrences X } \\
\hline Gr. gén. Déplacements & $\mathrm{Pe}$ & $\mathrm{Pi}$ & Tot. occ. & $\mathrm{Pe}$ & $\mathrm{Pi}$ & Tot. occ. & $\begin{array}{c}\text { Tot. occ. } \\
\mathrm{M}+\mathrm{X}\end{array}$ \\
\hline $\begin{array}{l}\text { 1. Circulation } \\
\text { a/ Véhicules de terre et leur } \\
\text { équipement } \\
\text { b/ Aviation, espace }\end{array}$ & $1 / 1$ & $2 / 2$ & 3 & $2 / 4$ & $4 / 6$ & 10 & 13 \\
$\begin{array}{l}\text { c/ Navigation } \\
\text { Groues / sous-groupes }\end{array}$ & $5 / 5$ & $1 / 1$ & 2 & $4 / 4$ & $1 / 1$ & 5 & 7 \\
\hline $\begin{array}{l}\text { 2. Déplacements } \\
\text { a/ Pourse déplacer } \\
\text { b/ L'action de se déplacer }\end{array}$ & $6 / 15$ & $5 / 18$ & 33 & $6 / 18$ & $5 / 20$ & 38 & 71 \\
\hline $\begin{array}{l}\text { 3. Pour faciliter le déplacement } \\
\text { (orientation, signalisation, vitesse) }\end{array}$ & $3 / 7$ & $7 / 17$ & 24 & $1 / 5$ & $7 / 13$ & 18 & 42 \\
\hline 4. / Faire/échouer & $4 / 12$ & $5 / 15$ & 27 & $7 / 17$ & $5 / 15$ & 32 & 59 \\
\hline $\begin{array}{l}\text { Nb total d'occurrences } \\
\text { Nb moyen d'occurrences / type }\end{array}$ & $\begin{array}{r}41 \\
2,0\end{array}$ & $\begin{array}{c}65 \\
2,4\end{array}$ & 106 & 52 & 61 & 113 & 219 \\
\hline
\end{tabular}

Tableau $2 a$

Le nombre de termes métaphorisants dans les groupes et sous-groupes sémiques du champ générique Déplacements. Leur répartition sur $\mathrm{Pe}$ et $\mathrm{Pi}$ dans $\mathrm{M}$ et $\mathrm{X}$

Les tableaux $2 a$ à $2 c$ indiquent un nombre à peu près égal de termes métaphorisants dans $M$ et $X, 348$ et 351 . Il faut cependant mettre ces chiffres en rapport avec la taille des rubriques examinées, présentée dans le tableau ci-dessous, où est aussi indiqué le pourcentage du nombre des mots dans $X$ par rapport à ceux de $M$, d'une part pour chacune des rubriques, d'autre part pour les deux rubriques mises ensemble. 


\begin{tabular}{|l|c|c|c|c|c|c|c|}
\hline & \multicolumn{2}{|c|}{ Types/occurrences M } & \multicolumn{4}{|c|}{ Types/occurrences X } \\
\hline Groupes sémiques & $\mathrm{Pe}$ & $\mathrm{Pi}$ & Tot. occ. & $\mathrm{Pe}$ & $\mathrm{Pi}$ & Tot. occ. & $\begin{array}{c}\text { Tot. occ. } \\
\text { M + X }\end{array}$ \\
\hline 1. Souffrir de / diagnostiquer maladie & $8 / 9$ & $8 / 12$ & 21 & $15 / 19$ & $13 / 22$ & 41 & 62 \\
\hline 2. Retour à la santé & $6 / 6$ & $8 / 13$ & 19 & $7 / 7$ & $7 / 10$ & 17 & 36 \\
\hline 3. Entrave au bon fonctionnement & $12 / 19$ & $10 / 19$ & 38 & $8 / 15$ & $11 / 25$ & 40 & 78 \\
\hline 4. Affaiblir / faire disparaître & $7 / 12$ & $3 / 7$ & 19 & $4 / 5$ & $2 / 2$ & 7 & 26 \\
\hline 5. Faire apparaître & $6 / 10$ & $3 / 9$ & 19 & $7 / 16$ & $4 / 6$ & 22 & 41 \\
\hline $\begin{array}{l}\text { Nb total d'occurrences } \\
\text { Nb moyen d'occurrences/type }\end{array}$ & 56 & 60 & 116 & 62 & 65 & 127 & 243 \\
\hline
\end{tabular}

Tableau $2 b$

Le nombre de termes métaphorisants dans les groupes et sous-groupes sémiques du champ générique Médecine.

Leur répartition sur $\mathrm{Pe}$ et $\mathrm{Pi}$ dans $\mathrm{M}$ et $\mathrm{X}$

\begin{tabular}{|c|c|c|c|c|c|c|c|}
\hline \multirow{2}{*}{$\begin{array}{l}\text { Ch. gén. Phénomènes naturels } \\
\text { Groupes sémiques }\end{array}$} & \multicolumn{3}{|c|}{ Types/occurrences M } & \multicolumn{4}{|c|}{ Types/occurrences X } \\
\hline & $\mathrm{Pe}$ & $\mathrm{Pi}$ & Tot. occ. & $\mathrm{Pe}$ & $\mathrm{Pi}$ & Tot. occ. & $\begin{array}{l}\text { Tot. occ. } \\
M+X\end{array}$ \\
\hline 1. Climat, météorologie, sinistres & $12 / 35$ & $12 / 23$ & 58 & $20 / 25$ & $12 / 19$ & 44 & 102 \\
\hline 2. Phénomènes célestes & $3 / 7$ & $4 / 6$ & 13 & $5 / 7$ & $7 / 7$ & 14 & 27 \\
\hline 3. Aquatique & $5 / 15$ & $3 / 9$ & 24 & $4 / 14$ & $5 / 10$ & 24 & 48 \\
\hline 4. Topographie & $7 / 11$ & $6 / 14$ & 25 & $3 / 4$ & $8 / 13$ & 17 & 42 \\
\hline 5. Végétal & $1 / 3$ & $3 / 3$ & 6 & $6 / 6$ & $5 / 6$ & 12 & 18 \\
\hline $\begin{array}{l}\mathrm{Nb} \text { total d'occurrences } \\
\mathrm{Nb} \text { moyen d'occurrences / type }\end{array}$ & $\begin{array}{l}71 \\
2,5\end{array}$ & $\begin{array}{c}55 \\
2\end{array}$ & 126 & $\begin{array}{l}56 \\
1,5\end{array}$ & $\begin{array}{l}55 \\
1,5\end{array}$ & 111 & 237 \\
\hline
\end{tabular}

Tableau 2c

Le nombre de termes métaphorisants dans les groupes et sous-groupes sémiques du champ générique Phénomènes naturels.

Leur répartition sur $\mathrm{Pe}$ et $\mathrm{Pi}$ dans $\mathrm{M}$ et $\mathrm{X}$

\begin{tabular}{|l|c|c|c|c|}
\hline Rubrique & Mots M & Mots X & Total & $\%$ X par rapport à M \\
\hline$P e$ & 128599 & 92577 & 221176 & $72 \%$ \\
\hline$P i$ & 167448 & 91857 & 259305 & $55 \%$ \\
\hline Total & 296047 & 184434 & 480481 & $62 \%$ \\
\hline
\end{tabular}

Tableau 3

Le nombre de mots des rubriques $P e$ et $P i$ dans $M$ et $X$ et le pourcentage des rubriques dans $X$ par rapport à celles du $M$ 
Le tableau 3 montre une différence considérable entre $M$ et $X$, surtout en ce qui concerne la rubrique $\mathrm{Pi}$, où la taille de $\mathrm{X}$ ne constitue qu'un peu plus de la moitié de celle de $M$. Compte tenu de ces relations, on peut conclure que le nombre relatif de termes métaphorisants est plus élevé dans $X$ que dans $M$, ce dernier produisant en moyenne un terme métaphorisant pour 848 mots et $X$ produisant en moyenne un terme métaphorisant pour 541 mots. En discutant des résultats affichés dans les tableaux $2 a$ à $2 c$, il faut toujours retenir ce fait. Même en nombres absolus, pour les types aussi bien que pour les occurrences, le plus souvent, les nombres de $X$ dépassent ou égalent ceux de $M$.

Dans l'aperçu suivant, nous traiterons d'abord de la rubrique Pe, ensuite de la rubrique $\mathrm{Pi}$.

\section{Les termes métaphorisants dans la rubrique Pe}

Le tableau za montre que $X$ a une fréquence d'occurrences bien plus élevée que $M$ en même temps que le rapport entre types et occurrences est très similaire entre les deux journaux : 2 occurrences/type dans $M$ et 2,2 occurrences/type dans $X$. Les choix lexicaux nous offrent, de part et d'autre, un stock de termes métaphorisants clichés, comme chemin, impasse, pont, route dans le sousgroupe sémique Pour se déplacer, feu vert dans le groupe sémique Pour faciliter le déplacement, et dérapage, dérive dans le groupe sémique Faire/échouer.

Malgré la lexicalisation manifeste d'une grande partie des termes métaphorisants rencontrés dans ce champ générique, nous trouvons des cas où un terme métaphorisant donne naissance à une métaphore filée ou à un défigement. Ces métaphores filées, souvent minimales, où seul un autre vocable reprend le terme métaphorisant en écho, sont plus fréquentes dans $X$ que dans M. Quelques exemples en sont chemin + larguer, route + barrer, impasse + sortir. D'autres sont plus élaborées, et l'une d'elles, particulièrement intéressante, laisse voir le processus créateur des métaphores en action :

Tout porte à croire que le balai des émissaires offrira, une fois encore à Oslo $/ l$, ce navire perclus de voies d'eau, un sursis. (X, Pe, 10 avril 1997, p. 86)

La première métaphore attendue dans cet exemple aurait plutôt été «le ballet des émissaires ». L'homonyme balai fait des émissaires une équipe de femmes de ménage essayant de mettre un peu d'ordre dans le chaos, métaphore peutêtre encore plus réussie. Quelle que soit l'explication - défigement d'une métaphore devenue cliché ou erreur d'un journaliste non familier avec le terme métaphorisant ballet -, la métaphore qui en résulte témoigne, d'une part, de notre capacité de trouver un sens à des jonctions inattendues de termes métaphorisants et de termes métaphorisés, d'autre part, du travail créateur du journaliste, et enfin, de «l'inventivité presque illimitée pour découvrir des analogies qui permettent d'utiliser un mot préexistant avec un sens nouveau, de l'appliquer à de nouveaux référents » (Baylon et Mignot, 1995, p. 92). 
Dans le champ générique Médecine, la relation entre types et occurrences est la même entre $M$ et $X$ que dans Déplacements, quoique à un niveau plus élevé: 39 types pour 56 occurrences dans $M$ et 41 types pour 62 occurrences dans X, c'est-à-dire respectivement 1,4 et 1,5 occurrences/type en moyenne. En ce qui concerne $M$, c'est le champ générique où les types sont de loin les plus nombreux. Il semblerait que le domaine de la santé et de la maladie, de la naissance et de la mort incite à la création d'une multitude de métaphores. Cette observation peut être rapprochée de celle de Svanlund (2001, p. 9), qui indique une figuralité forte des mots qui décrivent des expériences psychiques. Il se peut que les métaphores en rapport étroit avec le corps et l'esprit humain soient plus variées et plus vives, et cela peut-être parce que, ayant un besoin de trouver le mot fin, exact, pour décrire nos sensations physiques et psychiques, nous disposons d'un vocabulaire étendu et varié dans ce champ-là. Ce champ générique est aussi le seul à renfermer un nombre notable d'adjectifs métaphorisants. Ils sont particulièrement nombreux dans le groupe sémique Affaiblir / faire disparaître, groupe qui se démarque aussi par une forte prédominance de $M$ : il présente 12 occurrences, soit plus du double des 5 occurrences de X. La même relation entre types et occurrences que nous détectons dans le champ générique Médecine, 1,5, se retrouve dans le champ générique Phénomènes naturels pour $X$. En revanche, $M$ renferme très peu de types pour une multitude d'occurrences qui sont, elles, au nombre de 71 (tableau $2 \mathrm{c}$ ). Le rapport dans $M$ est de 2,5 occurrences/type, le plus grand écart dans notre matériau. Selon le tableau 2c, le groupe sémique Climat, météorologie, sinistres affiche un nombre particulièrement élevé d'occurrences en même temps qu'un nombre restreint de types. Trois termes métaphorisants dominent: climat, gel/geler et vent. Ces termes métaphorisants sont utilisés dans $\mathrm{X}$ aussi, mais accompagnés de nombreux exemples où apparaissent des termes métaphorisants dénotant des phénomènes météorologiques, volontiers des sinistres : avalanche, érodé, séisme, tremblement de terre, etc. Le peu de variation de termes métaphorisants a aussi pour conséquence, dans $M$, une monotonie syntaxique, un même terme métaphorisant ayant tendance à s'insérer dans des syntagmes identiques : climat + adjectif épithète/de N, gel + de N.

\section{Les termes métaphorisants dans la rubrique Pi}

De nouveau, nous voyons que la seule différence notable dans la relation entre types et occurrences dans $M$ et $X$ se situe dans le champ générique Phénomènes naturels, où $\mathrm{M}$ a nettement plus d'occurrences par type (2) que X $(1,5)$. Ce champ générique diffère encore des deux autres en ce que les occurrences, en nombres absolus, sont moins nombreuses sous Pi que sous Pe.

En général, les termes métaphorisants ne se distribuent pas différemment sous les deux rubriques, ni dans $M$, ni dans $X$. Un seul groupe sémique dans le 
champ générique Déplacements attire l'attention, à savoir Pour faciliter le déplacement, où le nombre d'occurrences est bien plus élevé sous Pi. Pour la plupart, les termes métaphorisants sont les mêmes que sous Pe mais utilisés en plus grand nombre.

En ce qui concerne le champ générique Médecine, les différences entre Pe et Pi sont plus nombreuses. Ainsi, le groupe sémique Entrave au bon fonctionnement est plus fréquent sous $\mathrm{Pi}$ dans $\mathrm{X}$, tandis que le groupe sémique Affaiblir / faire disparaître y est très faiblement représenté. Dans le groupe sémique Retour à la santé, c'est dans $\mathrm{M}$ qu'on voit une fréquence nettement plus élevée sous Pi que sous Pe. Le terme métaphorisant dégraisser/dégraissage est utilisé, dans $M$ et $X$, uniquement en parlant du système scolaire. Il serait intéressant de suivre le développement, au cours des années, de ce terme métaphorisant.

Terminons ce petit tour d'horizon fréquentiel par un coup d'œil sur le champ générique Phénomènes naturels. Comme nous l'avons déjà fait remarquer, les occurrences sont moins nombreuses dans $M$ par rapport à la rubrique $P$ e. Cela est dû principalement à l'emploi moins fréquent des termes métaphorisants climat, gel/geler et vent dans le groupe sémique Climat, météorologie, sinistres. Notons, par ailleurs, que $\mathrm{X}$ augmente considérablement l'emploi des termes métaphorisants relevant du groupe sémique Topographie. Comme le montre le tableau $2 \mathrm{c}$, cette augmentation concerne les types aussi bien que les occurrences.

Les commentaires ci-dessus laissent comprendre que dans chaque champ générique, un certain nombre de termes métaphorisants reviennent fréquemment. Ont-ils également tendance à se joindre aux mêmes termes métaphorisés? C'est de cette question que nous traiterons dans la section suivante.

\section{La sélection des termes métaphorisés}

En ce qui concerne les catégories de termes métaphorisés, Abstrait, Immatériel, Collectif, Concret et Humain qui, en conjonction avec les termes métaphorisants, créent les métaphores combinatoires, il s'avère que deux de ces catégories, Concret et Humain, sont quasi absentes de notre matériau. Un examen de la répartition des termes métaphorisés montre qu'ils entrent très majoritairement dans des jonctions conventionnelles, créant des figements sémantiques, syntaxiques, voire lexicaux.

Dans $M$, les termes métaphorisés Collectivité ne sont qu'au nombre de 4 pour le champ générique Phénomènes naturels et ils ne sont que 12 dans $X$, alors qu'ils sont entre 20 et 30 pour chacun des champs génériques Déplacements et Médecine. Par ailleurs, nous retrouvons le nombre le plus élevé de termes métaphorisés Abstrait dans le champ générique Phénomènes naturels. Sans qu'on puisse dire qu'il y a une affinité entre, par exemple, climat et idées ou états mentaux divers, cette lexie, quand elle apparaît en métaphore, fait 
naturellement évoquer des notions comme atmosphère et ambiance. Ces associations créent, dans nos exemples, des métaphores où les termes métaphorisés parlent d'amitié, de pessimisme, de tension, de sérénité, etc. Avec climat, l'exemple le plus évident, aux métaphores les plus nombreuses, les termes métaphorisants racine, vague, horizon, séisme sont ceux qui se joignent de préférence aux termes métaphorisés abstraits.

Les régularités sémantico-lexicales sont accompagnées de régularités syntaxiques créant un grand nombre de métaphores de l'ordre terme métaphorisant substantif + terme métaphorisé épithète ou de N:

D’où le surnom « minister for meddling » [...] qui a été donné à l'un de ceux qui symbolisent le Labour nouveau mais dont les racines travaillistes sont anciennes. (M, Pe, 30 septembre 1997, p. 2)

Dans les autres champs génériques, les termes métaphorisés abstraits sont moins nombreux et leur sélection de termes métaphorisants moins uniforme, à l'exception du terme métaphorisant fracture, où c'est la métaphore - éphémère? - fracture sociale qui réapparaît dans $M$ aussi bien que dans $X$. Récurrente sous la rubrique $\mathrm{Pi}$, elle indique que c'est plutôt là qu'on traite de la problématique des différences sociales:

Meaux [...] est devenue un laboratoire de plâtres pour fracture sociale. (X, Pi, 10 avril 1997, p. 36)

Nous considérons la métaphore fracture sociale, dans cet exemple, comme une métaphore noyau, filée par laboratoire de plâtres.

La catégorie Immatériel renferme, sous les rubriques $\mathrm{Pe}$ et $\mathrm{Pi}$, principalement des termes métaphorisés qui sont à ramener à des objets ou à des actions concrets. Dans chaque champ générique, une majorité des métaphores ont une tonalité négative. Cela ressort clairement dans les métaphores aux termes métaphorisants impasse, capoter, moribond, exsangue, écueil, maquis, où le sémantisme négatif prédomine, mais même des termes métaphorisants comme freiner et geler s'insèrent souvent dans un cadre négatif. Si freiner et geler n'ont pas un sens a priori entièrement négatif, le discours où sont insérées les métaphores exprime en règle générale des procès ou des résultats non désirés. À titre d'illustration, voici un exemple :

La crise politique en Inde risque de freiner les réformes économiques. (M, Pe, 2 avril 1997, p. 3)

On touche ici à un champ de recherche qui se penche sur l'implicite des énoncés, sur la question des opinions exprimées à travers le discours. De plus, l'interprétation positive ou négative d'un énoncé comme celui de l'exemple ci-dessus dépend, d'un côté, du lecteur individuel et, de l'autre, de l'environnement politique, social et historique où apparaît l'énoncé. 
La jonction d'un terme métaphorisant qui rend négatif ce qui est représenté par le terme métaphorisé - des termes métaphorisés récurrents sont, entre autres, projet, pacte, relations diplomatiques/économiques, processus de paix - donne à ce groupe de métaphores un ton pessimiste.

Comme on devait s'y attendre, ce sont les collectivités politiques et administratives qui se concentrent sous les rubriques Pe et Pi avec une petite majorité pour la première. Parmi les termes métaphorisés du groupe Collectivité qui se rangent dans la catégorie politique et administrative, nous avons mis, entre autres, les pays, les villes, les partis et organisations politiques, judiciaires, scolaires et militaires. Même en termes absolus, $X$ renferme un nombre plus élevé d'exemples de ce groupe -62 , contre les 58 exemples relevés dans $M$. Le champ générique Médecine faisant naître la majorité de ces métaphores, le contraste avec le champ générique Phénomènes naturels est spectaculaire: nous n'y avons repéré que 16 exemples contre 61 sous Médecine, les chiffres valant pour $M$ et $X$ confondus. À notre sens, cela confirme encore notre hypothèse proposant des utilisations conventionnelles d'un grand nombre de métaphores, et pas seulement de leur partie métaphorisante.

Un regard sur les métaphores du champ générique Médecine montre que les deux tiers de celles retrouvées dans $X$ et plus de la moitié dans $M$ relèvent de deux groupes sémiques, Souffrir de/diagnostiquer maladie et Entrave au bon fonctionnement. Une multitude d'exemples parlent de l'abcès, de l'hypertrophie, de l'asphyxie/étouffement, de la paralysie des collectivités, créant des métaphores à sémantisme négatif, et cela dans les deux journaux. La même observation vaut pour bon nombre de termes métaphorisants des autres champs génériques, par exemple dérive du champ générique Déplacements, qui se joint, dans $M$, à peu près exclusivement à des termes métaphorisés tels que régime, parti conservateur et organisation séparatiste. Comme ces termes métaphorisés figurent surtout sous la rubrique Pe, cela veut dire que $M$ nous présente un grand nombre de métaphores à sémantisme négatif sous cette rubrique, quand il s'agit du groupe de termes métaphorisés Collectivité, ce qui refléterait une tendance de ce journal à s'occuper, lorsqu'il traite des questions internationales, de problèmes et d'événements ayant des implications négatives. Il y a aussi la possibilité que la variation des termes métaphorisants négatifs soit plus grande que celle des termes métaphorisants au sémantisme positif, peut-être enfin une tendance à dramatiser ce qui est déjà dramatique en utilisant plus de métaphores qu'on ne le fait en écrivant sur les sujets «positifs ». Enfin, les articles sous cette rubrique traitent plutôt des grands problèmes internationaux. 


\section{Le cas des métaphores pragmatiques}

Les métaphores pragmatiques constituent un groupe important sous les rubriques Pe et Pi. Ainsi, dans chacun des champs génériques Déplacements et Phénomènes naturels, elles forment le groupe le plus grand dans les deux journaux, ce qui est aussi le cas pour $X$ dans le champ générique Médecine. Ce dernier journal se sert donc, encore plus amplement que $M$, de ce luxe du lexique ${ }^{8}$. Tous champs génériques confondus, les métaphores pragmatiques sont plus fréquentes sous $\mathrm{Pi}$, d'une manière plus prononcée pour Déplacements dans $\mathrm{M}$ et Médecine dans $\mathrm{X}$.

Les métaphores pragmatiques comportent beaucoup de métaphores plurilexicales, entre autres des locutions comme mener en bateau, embrasser pour mieux étouffer, sentir l'orage. Ces locutions métaphoriques sont particulièrement nombreuses dans le champ générique Déplacements, où elles sont aussi, dans bien des cas, filées:

Je marche sur des œufs, reconnaît pour sa part Jean-Marie Bockel, on est sur un chemin de crête avec, d'un côté, un certain angélisme à gauche, de l'autre des dérapages à droite. (M, Pi, 15 janvier 1998, p. 10)

La locution métaphorique marcher sur des œufs incite à filer la métaphore dans le même champ générique, produisant ainsi une métaphore mixte, combinatoire et pragmatique, très élaborée. Justement le terme métaphorisant chemin, avec un petit nombre d'autres lexies du même champ générique, par exemple feu vert, route, dérive, impasse, produit un nombre élevé de métaphores pragmatiques.

Comment expliquer la présence plus importante des métaphores pragmatiques sous la rubrique Pi? Est-ce que le fait ou le sentiment d'écrire sur des sujets plus proches, plus familiers aux lecteurs incite à un style plus décontracté ? Est-ce un moyen de rendre plus «humains », plus «communs » les politiques dont on rapporte les faits et gestes ? Peut-être une tendance à se servir de plus de métaphores en traitant de personnes plutôt que de sujets abstraits? Toujours est-il que beaucoup de ces métaphores sont employées en parlant de personnes.

Les métaphores pragmatiques jouent un rôle important pour introduire, expliciter ou résumer un discours. Cette dernière fonction se réalise par l'usage anaphorique de la métaphore:

Devant une telle accumulation d'impasses, il est permis de se demander si la seule vraie politique de Netanyahu ne serait pas la seule fuite en avant vers le néant. syndrome suicidaire, là encore, rappelle une certaine politique arabe avant Sadate et Camp David. (X, Pe, 2 octobre 1997, p. 103)

8. Tournure que nous empruntons à Schapira (1999, p. 20). 
L'anaphore métaphorique devient simultanément un moyen important pour éviter des répétitions fastidieuses et, comme le montre l'exemple ci-dessus, une caractéristique de l'événement ou du référent visés par l'anaphore. L'emploi pragmatique des termes métaphorisants conventionnels souligne leur autonomie et pose la question des frontières entre métaphore, catachrèse et polysémie.

Notre inventaire montre que $X$ a une fréquence plus élevée de métaphores que $M$, mais aussi qu'une part considérable des termes métaphorisants est commune à ces deux journaux. La division en groupes sémiques des champs génériques examinés nous a aidée à étudier de plus près, d'une part, les différences entre $M$ et $X$, d'autre part, les différences entre Pe et $P i$. Il s'avère que le nombre de métaphores est presque le même dans les deux journaux, mais comme le corpus de $X$ ne renferme qu'environ $60 \%$ du nombre des mots de $M$, la fréquence relative des métaphores du premier journal est bien plus élevée. Nous constatons aussi que les métaphores sont plus nombreuses sous la rubrique $\mathrm{Pi}$ que sous la rubrique Pe dans les deux champs génériques Déplacements et Médecine, tandis que le champ générique Phénomènes naturels présente une forte prédominance pour Pe dans $M$ et des rapports égaux dans X. C'est sous Pe dans $M$ aussi que le nombre de types est le plus réduit.

Quant aux groupes sémiques, certains d'entre eux sont favorisés par les deux journaux, soit sous Pe, soit - beaucoup plus souvent - sous Pi. Ainsi, le groupe sémique Pour faciliter la circulation renferme deux à trois fois plus d'occurrences sous Pi que sous Pe. D'autres fois - le groupe sémique Végétal en est un exemple très net $-X$ utilise, même en nombres absolus, le double des occurrences de $M$.

L'observation des termes métaphorisés indique que les trois catégories $A b$ strait, Immatériel et Collectivité se répartissent différemment selon le champ générique. Ainsi, les termes métaphorisés de la catégorie Collectivité sont rares dans le champ générique Phénomènes naturels alors que les termes métaphorisés de la catégorie Abstrait y sont très fréquents. Cette observation confirme notre hypothèse: ce ne sont pas seulement un grand nombre de termes métaphorisants qui sont récurrents dans notre matériau, mais bon nombre de métaphores combinatoires. Cela est vrai aux niveaux sémantique, syntaxique et lexical. Il est aussi intéressant de constater que la jonction des termes métaphorisants et des termes métaphorisés aboutit plus souvent que l'inverse à des métaphores dont le sémantisme est négatif.

Les métaphores pragmatiques, particulièrement fréquentes dans $X$, raniment le style mais ne sont pas qu'un luxe du lexique. Dans cet article, nous avons mis en lumière leur fonction anaphorique. Dans leur ensemble, métaphores combinatoires et métaphores pragmatiques sont un moyen important 
de la prose journalistique pour mettre en relief les faits, résumer un débat. Elles aident à créer un cadre familier, caractéristique de ce genre.

\section{Références}

BAYLON C., MIGNOTX., 1995, Sémantique du langage. Initiation, Paris, Nathan.

CHARBONNEL N., KLEIBER G., 1999, La métaphore entre philosophie et rhétorique, Paris, PUF.

DÉTRIE C., 2001, Du sens dans le processus métaphorique, Paris, Honoré Champion.

ENGWALL G., BARTNING I., 1989, "Le COSTO. Description d'un corpus journalistique », Moderna språk, $n^{\circ} 83, p .343-348$.

FERRARI S., 1997, "Méthode et outils informatiques pour le traitement des métaphores dans les documents écrits », thèse de doctorat, LIMSI-CNRS, université Paris-Sud, Orsay.

FONTANIER P., 1977 [1830], Les figures du discours, Paris, Flammarion.

GREVISSE M., 1993, Le bon usage, Louvain-la-Neuve, Duculot.

HALLYN F., 1994, "De la métaphore filée au modèle analogique : cohérence et cohésion », Travaux de linguistique, $\mathrm{n}^{\circ}$ 29, p. 107-123.

KERZAZI-LASRI R., 2003, La métaphore dans le commentaire politique, Paris, L'Harmat$\tan$.

MALMBORG (von) G., 2004, "La spécificité de la métaphore journalistique: les métaphores de quelques champs génériques dans Le Monde et L'Express. Analyses thématique, comparative et distributive ", Cahiers de la recherche, $n^{\circ} 25$, thèse de doctorat, université de Stockholm, département de français et d'italien.

PAPPAS C., 2003, « Metaforer i det politiska språket: En studie av de socialdemokratiska och de konservativ-liberala partiledarnas retorik i Grekland och Sverige vid millennieskiftet », Dissertationes Neohellenicae, Universitatis Stockholmiensis, Institutionen för klassiska språk 2003.

PRANDI M., 1992, Grammaire philosophique des tropes, Paris, Minuit.

RIEGEL M., PELLAT J.-C., RIOUL R., 1994, Grammaire méthodique du français, Paris, PUF.

ROBERT P., 1996, Le Nouveau Petit Robert. Dictionnaire alphabétique et analogique de la langue française, Paris, Dictionnaires Le Robert.

SCHAPIRA C., 1999, Les stéréotypes en français. Proverbes et autres formules, Paris, Ophrys.

SCHMITT C., 1988, « Gemeinsprache und Fachsprache im heutigen Französisch. Formen und Funktionen der Metaphorik in wirtschaftsfachsprachlichen Texten », H. Kalverkämpfer éd., Fachsprachen in der Romania, p. 113-129.

SULLET-NYLANDER F., 1998, "Le titre de presse. Analyses syntaxique, pragmatique et rhétorique », Cahiers de la recherche, $\mathrm{n}^{\circ} 8$, thèse de doctorat, université de Stockholm, département de français et d'italien.

SVANLUND J., 2001, Metaforen som konvention. Graden av bildlighet i svenskans vikt- och tyngdmetaforer, Stockholm, Almqvist et Wiksell International.

VILLARD M., 1984, Les universaux métaphoriques, Berne, Peter Lang. 